\title{
The LLE, pattern formation and a novel coherent source ${ }^{\star}$
}

\author{
Fabrizio Castelli ${ }^{1}$, Massimo Brambilla ${ }^{2}$, Alessandra Gatti ${ }^{3,4}$, Franco Prati ${ }^{4,5}$, and Luigi A. Lugiato ${ }^{5}$ \\ 1 Dipartimento di Fisica, Università degli Studi di Milano and INFN Sezione di Milano, via Celoria 16, 20133 Milano, Italy \\ 2 Dipartimento di Fisica Interateneo and CNR-IFN, Università e Politecnico di Bari, via Amendola 173, 70123 Bari, Italy \\ 3 Istituto di Fotonica e Nanotecnologie del CNR, Piazza Leonardo da Vinci 32, Milano, Italy \\ 4 CNISM, Research Unit of Como, via Valleggio 11, 22100 Como, Italy \\ ${ }_{5}^{5}$ Dipartimento di Scienza e Alta Tecnologia, Università dell'Insubria, via Valleggio 11, 22100 Como, Italy
}

Received 2 December 2016

Published online (Inserted Later) - (c) EDP Sciences, Società Italiana di Fisica, Springer-Verlag 2017

\begin{abstract}
The LLE was introduced in order to provide a paradigmatic model for spontaneous spatial pattern formation in the field of nonlinear optics. In the first part of this paper we describe in details its historical evolution. We underline, first of all, that the multimode instability of optical bistability represents an important precursor of the LLE. Next, we illustrate how the original LLE was conceived in order to describe pattern formation in the planes transverse with respect to the longitudinal direction of propagation of light in the nonlinear medium contained in the optical cavity. We emphasize, in particular, the crucial role of the low transmission limit (also called mean field limit or uniform field limit in the literature) in determining the simplicity of the equation. In discussing transverse pattern formation in the LLE, we underline incidentally the presence of very important quantum aspects related to squeezing of quantum fluctuations and to quantum imaging. We consider not only the case of global patterns but also localized structures (cavity solitons and their control). Then we turn to the temporal/longitudinal version of the LLE, formulated by Haelterman, Trillo and Wabnitz, and to its equivalence with the transverse LLE in $1 \mathrm{D}$, discussing especially the phenomenon of temporal cavity solitons, their experimental observation and their control. Finally for the first part we turn to the very recent topic of broadband frequency combs, observed in a versatile multiwavelength coherent source (driven Kerr microcavity), which is raising a lot of interest and of research activities because of its very favourable physical characteristics, which support quite promising applicative perspectives. Kerr microcavities realize in an ideal manner the basic assumptions of the LLE, and the spontaneous formation of travelling patterns along the microcavity is the crucial mechanism which creates the combs and governs their features. Thus the LLE represents a case of spontaneous pattern formation which is immediately linked to a promising applicative avenue. The second part of the paper is devoted to the detailed derivation from the Maxwell-Bloch equations of the temporal/longitudinal LLE which was proposed by ourselves many years ago without providing a complete derivation. Such an equation is equivalent to the standard temporal/longitudinal version of the LLE in the case of anomalous dispersion. Our derivation elucidates in the best way the connection between the temporal/longitudinal version of the LLE and the multimode instability of optical bistability.
\end{abstract}

\section{Introduction}

This article concerns the equation, proposed by one of us and R. Lefever nearly thirty years ago [1], that in the following we call LLE. From a mathematical standpoint, it can be defined as a driven, damped and detuned nonlinear Schroedinger equation. With respect to commonly used equations such as, for example, the Ginzburg-Landau equation, a distinctive feature of the LLE is represented by the inhomogeneous driving term, which discloses a universe of physical effects.

* Contribution to the Topical Issue "Theory and applications of the Lugiato-Lefever Equation", edited by Yanne K. Chembo, Damia Gomila, Mustapha Tlidi, Curtis R. Menyuk.

a e-mail: franco.prati@uninsubria.it
The original aim of the LLE was to provide a paradigm for pattern formation á la Turing [2] in nonlinear optical systems. Phenomena of spontaneous pattern formation, both of spatial and temporal nature, are ubiquitous in the vast domain of nonlinear dynamical systems, encompassing e.g. hydrodynamics, chemistry, biology, population dynamics, social sciences. General disciplines such as Haken's synergetics [3] or Prigogine's theory of dissipative structures [4] have tried to unify this field and to identify some general principles that govern these phenomena. As already underlined in 1994 [5], the case of optics presents two special features that are interesting and stimulating in this connection. First, optical systems are fast and have a large frequency bandwidth, therefore they lend themselves naturally to applicative perspectives, for instance in 
telecommunications and information processing. The second relevant feature is that optical systems display interesting quantum effects at room temperature and therefore they can play an important role in quantum technologies.

The model [1] was constructed by following a criterion of simplicity, which led to the selection of a cubic Kerr nonlinearity, of an optical ring cavity driven by a cw coherent input field and of conditions that, in the stationary states, ensure the uniformity of the electric field envelope along the cavity. As it is well known, the Kerr nonlinearity in a cavity leads to a dominant bistable scenario [6]. The combination of nonlinearity and diffraction gives rise to a variety of $1 \mathrm{D}$ and $2 \mathrm{D}$ patterns, and to cavity solitons, in the transverse planes orthogonal to the longitudinal direction of propagation.

Five years later Haelterman, Trillo and Wabnitz [7] formulated the temporal/longitudinal version of the LLE in which, basically, diffraction is replaced by group velocity dispersion or, from a mathematical viewpoint, the transverse Laplacian with respect to the transverse spatial variables is replaced by the second derivative with respect to the retarded time in the cavity. Even if the LLE in [7] is mathematically fully equivalent to the LLE in [1] in $1 \mathrm{D}$, the physical conditions are complementary because in the case of [7] the electric field envelope is uniform in the transverse plane, whereas it develops patterns and cavity solitons in the longitudinal direction. Such patterns propagate along the cavity with the light velocity (therefore they are longitudinal/temporal patterns) and, in the output of the cavity, they generate a periodic train of pulses.

In this way, the LLE unifies spatial phenomena, that arise in the transverse planes, with spatio-temporal phenomena that occur in the longitudinal direction.

On the other hand, the temporal/longitudinal version of the LLE is naturally linked to works by Bonifacio and one of us, appeared well before the LLE itself, which predicted the same kind of phenomena in the framework of the multimode instability in the two-level model of optical bistability $[8,9]$. The advantage of the LLE is, however, that it identifies conditions in which such phenomena are by far more accessible experimentally and display features much richer and promising for applicative perspectives.

Frequency combs are sets of equidistant frequency lines in short-pulse mode-locked lasers. Their development by Hall [10] and Haensch [11] revolutionized the measurement of frequencies and opened out a vast scenario of applications in fundamental and applied physics. Recently Kippenberg et al. demonstrated the realization of broadband frequency combs using the whispering gallery modes in high- $Q$ microresonators containing Kerr media [12]. The generation of such Kerr frequency combs occurs from the four-wave-mixing (FWM) processes activated by the interaction between the monochromatic driving field, injected with a frequency resonant or nearly resonant with a cavity mode, and the Kerr medium. Microresonator Kerr frequency combs are foreseen to have a strong impact as a compact, low cost, low-power, chip compatible technology, which has stimulated a considerable worldwide effort in this approach.
Chembo [13,14], Coen [15,16], and Matsko [17] (in alphabetical order) with their collaborators have demonstrated that the LLE (or its generalizations which include higher order dispersion terms) is the appropriate model for the description of Kerr comb generation and can be conveniently utilized to explore and predict the comb characteristics as a function of the system parameters. From the spatio-temporal viewpoint, the spontaneous formation of travelling patterns along the cavity, described by the LLE, is the crucial mechanism which creates the combs and governs their features. The spectacular technological progress in the field of photonics, leading to the discovery of Kerr frequency combs, has implicitly realized all the rather idealized conditions assumed in the formulation of the LLE.

Since the seventies, it is well known that a strong signal field which saturates a two-level medium can induce gain in a weak probe beam with a frequency different from that of the signal field [18-20]. This concept is at the root of the multimode instability of optical bistability. Even if the system is passive, the strong driving field can induce gain in sidemodes of the resonant mode, and this gain originates the instability, the traveling pattern and the pulsed output $[8,9,21]$. Thus, with respect to the sidemodes the system behaves as active, i.e. as a source.

The parametric conditions considered in $[8,9]$ are unfavourable from an experimental viewpoint and give rise to narrowband frequency combs. Instead, Kerr frequency combs as those generated in [12] and in many other experiments (see e.g. [22-25]) are broadband and can arrive at spanning an octave. Thus, the systems which generate such combs can be regarded as novel coherent multiwavelength sources, where all the lines, with the exception of the central line corresponding to the driving frequency, are created by the gain induced by the FWM processes. Experimentally observed combs are compared with the predictions of the LLE in $[15,16,26,27]$. In [26] universal scaling laws of Kerr frequency combs are derived from the LLE.

The investigations in the vast area of pattern formation, theoretical and experimental, have typically been of purely fundamental character. The case of the LLE is special because it is intimately linked to the realization of a versatile multiwavelength coherent source, that brings important promises also to applied physics, especially to ultradense optical fiber networks, because it provides several independent but frequency locked subcarriers that can be controlled precisely and individually. Each element of the comb can be utilized as carrier for coherent data transmission at long distance, with quite promising characteristics $[28,29]$. A review of the field of Kerr combs, which includes a discussion of applicative perspectives, can be found in [30].

The aim of this article is twofold. The first is to describe, in Section 2, the history centered around the LLE. Many points have been already discussed in the introduction, but in Section 2 we add all the necessary details.

The second aim arises from the fact that several years ago the same authors of the present article formulated [31] a longitudinal version of the LLE which is equivalent to 
that introduced in [7] (in the case of anomalous dispersion) and includes the group velocity dispersion term, but is derived from the multimode two-level model in the limit of large atomic detuning, which in turn implies the cubic approximation. In [31] such a longitudinal model was described as obtained from the direct generalization of the derivation of the three-mode model given in [32], but its detailed derivation was not provided there. We describe it here, in Section 3, especially because it elucidates in the best way the connection between the tempo$\mathrm{ral} /$ longitudinal version of the LLE, introduced in [7], and the multimode instability of two-level optical bistability. Some conclusions are drawn in Section 4.

In the following, for uniformity of notations and of procedures we will systematically refer to the treatment of the book [21].

\section{The history around the LLE}

\subsection{Pre-history}

The search for the multimode instability of optical bistability [8,9] (see also pages 291-296 in Ref. [21]) was inspired by the multimode laser instability discovered by Risken and Nummedal [33] and Graham and Haken [34], but in this case the instability arises in a passive driven system, which represents a totally different physical context.

The model describes a system of two-level atoms contained in a ring cavity and driven by a coherent, monochromatic, stationary field injected into the cavity. As a consequence of the instability, a periodic pattern forms in the slowly varying envelope of the electric field travelling along the cavity and generates, in the output, a regular train of pulses (self-pulsing). Thus, the system works as a converter of cw light to pulsed light [35]. The instability was first predicted under conditions of exact resonance between the frequency of the input field, a cavity frequency and the atomic transition frequency $[8,9]$, and was then extended to the detuned configurations [36].

For the parametric ranges examined in $[8,9,35,36]$ the rise of the instability requires a long cavity. The first experimental observation of this phenomenon was obtained by Segard and Macke under detuned conditions using a folded 182-m long cavity operated in the microwave regime [37]. The frequency comb in the output displayed four peaks around the central one (Fig. 1).

\subsection{The LLE and transverse spatial patterns}

The LLE was conceived with the aim of providing, in the framework of optics, a model which could play the same paradigmatic role as the Prigogine-Lefever model [38], usually called Brusselator, in nonlinear chemical reactions. The latter model consists in two coupled nonlinear equations which govern the interaction of two reactants in an open environment. The formation of Turing patterns is

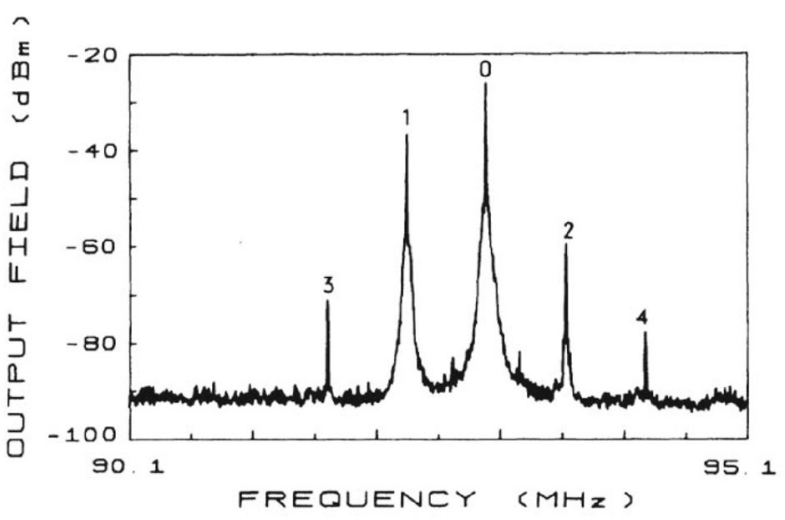

Fig. 1. Frequency comb observed in the multimode instability of optical bistability [37]. The different peaks correspond to field frequencies equal to (0) $\nu_{0}=\omega_{0} / 2 \pi$ input field frequency, (1) $\nu_{0}-\nu_{\mathrm{sp}}$, (2) $\nu_{0}+\nu_{\mathrm{sp}}$, (3) $\nu_{0}-2 \nu_{\mathrm{sp}}$, (4) $\nu_{0}+2 \nu_{\mathrm{sp}}$, where $\nu_{\mathrm{sp}}$ is the frequency of the spontaneous oscillations in the output intensity, which arise from the instability. Reprinted figure from reference [37], with permission by American Physical Society.

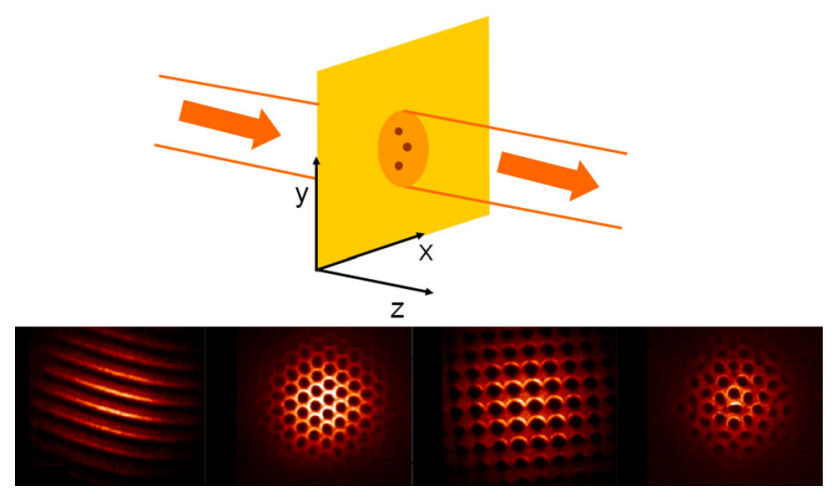

Fig. 2. Top: a transverse pattern may arise when a broad section coherent beam interacts with a nonlinear medium. Bottom: example of patterns observed in $\mathrm{Na}$ vapor by Lange, Ackemann et al.

induced by the interplay of the nonlinearity with the diffusion of the reactants. The pattern formation occurs in $2 \mathrm{D}$, i.e. in a "large aspect ratio" configuration in which the system is contained in a vessel that is large in the spatial directions $x$ and $y$ and thin in the third direction $z$, so that the variable $z$ does not appear in the model.

In the case of optics, the role of diffusion is played by diffraction and the coordinates $x$ and $y$ are those which span the planes orthogonal to the longitudinal direction $z$ along which the light propagates (see Fig. 2). In the paraxial approximation, diffraction is described by a term proportional to the transverse Laplacian of the electric field envelope, exactly as diffusion is described by terms proportional to the transverse Laplacian of the concentration of the reactants. A basic difference is that in the case of diffraction the Laplacian is multiplied by the imaginary unit. The field envelope $E(x, y, z, t)$ is related to the electric field (assumed linearly polarized for simplicity) 
$1 \mathcal{E}(x, y, z, t)$ in the following way

$$
\mathcal{E}(x, y, z, t)=\frac{1}{2} E(x, y, z, t) \exp \left[-i \omega_{0}(t-z / c)\right]+\text { c.c. },
$$

where $\omega_{0}$ is the frequency of the input field.

On the other hand, by looking at Figure 2 one realizes that in general optical systems are far from having a large aspect ratio, because the laser field which interacts with the nonlinear medium propagates along it in the longitudinal direction $z$ and therefore the $z$ variable cannot be ignored in general. A necessary step to solve this problem is to consider a configuration in which the nonlinear medium is contained in an optical cavity. In the following we consider a ring cavity with planar mirrors for definiteness.

In the description of a two-level system interacting with a coherent field within the cavity, the key elements are the Maxwell-Bloch equations (see Sect. 4.4 in Ref. [21]) and the field boundary condition in the ring cavity introduced in [39] (see Eq. (8.36) in Ref. [21]). Such a condition introduces a basic characteristic time, i.e. the cavity roundtrip time $\mathcal{L} / \tilde{c}$, where $\mathcal{L}$ is the cavity length and $\tilde{c}$ is the light velocity in the material, and corresponds to the inverse of the free spectral range (apart from a factor $2 \pi)$. For the sake of simplicity, we assume that the length of the sample is equal to the cavity length, i.e. the material fills the whole cavity.

In the rate equation limit, Ikeda [40] converted the Maxwell-Bloch equations and their boundary condition into a set of difference-differential equations and, in appropriate parametric conditions, into a set of difference equations (map) which govern the evolution of the field envelope and of an appropriate auxiliary variable at each roundtrip. The main virtue of this procedure is that it led to predicting the possibility of optical chaos in optical bistability [40]. The first models, which were used to describe transverse pattern formation in optical systems [41], were a generalization of the Ikeda procedure to include diffraction, but far from the simplicity of the Brusselator.

The limitation of the map approach is that it fails to identify the second basic characteristic time of the field envelope, i.e. the cavity decay time (or lifetime of photons in the cavity) $\mathcal{L} / \tilde{c} T$, where $T$ is the intensity transmissivity coefficient of the input and output mirrors of the cavity. Such a temporal scale emerges as soon as the mirror transmissivity becomes small. The cavity decay time corresponds to the inverse of the cavity linewidth.

The limit which allows to capture the advantages linked to the second characteristic time is the so-called low transmission limit (also called mean field limit or uniform field limit in the literature) first introduced in [39]. This is the following multiple limit

$$
T \ll 1, \quad \alpha^{\prime} \mathcal{L} \ll 1 \quad \text { with } \quad C=\frac{\alpha^{\prime} \mathcal{L}}{2 T} \quad \text { arbitrary },
$$

where $\alpha^{\prime}$ is the field absorption coefficient, $C$ is the bistability parameter, and

$$
\left|\delta_{0}\right|=\frac{\left|\omega_{c}-\omega_{0}\right|}{\tilde{c} / \mathcal{L}} \ll 1 \quad \text { with } \quad \theta=\frac{\delta_{0}}{T} \quad \text { arbitrary, }
$$

where $\omega_{0}$ is the frequency of the input field which is injected into the cavity and $\omega_{c}$ is the cavity frequency closest to $\omega_{0}$. Condition (2) states that in a single pass through the atomic medium the field envelope undergoes a negligible variation but, since the lifetime of photons in the cavity corresponds to several roundtrips because $T \ll 1$, the field envelope undergoes a sizable variation over the long time scale $\mathcal{L} / \tilde{c} T$. On the other hand condition (3) states that the frequency difference between the resonant cavity frequency and the input frequency is small with respect to the free spectral range and on the order of the cavity linewidth. Condition $\alpha^{\prime} \mathcal{L} \ll 1$ can be realized either using a short cavity or a weak nonlinearity. Condition $T \ll 1$ implies that the cavity is high- $Q$.

In the low transmission limit the Maxwell-Bloch equations are conveniently rephrased in the form of equation (16) which appear in the following of this paper (see also Sect. 12.2 in Ref. [21]) and the field boundary condition in the ring cavity reduces to a periodic boundary condition (see Sect. 12.1 in Ref. [21]).

If one assumes, in addition to conditions (2), (3), that only the resonant cavity mode has a nonzero amplitude (singlemode limit), one has that the field envelope is uniform along the cavity, so that the field envelope varies only with respect to time (with the temporal scale of the cavity decay time) and to the transverse variables $x$ and $y$ (see Fig. 1), and this feature makes it possible to formulate a model for transverse optical pattern formation with the same level of simplicity as the Brusselator. In order to achieve this, the model must involve only the field envelope, which is a complex variable, so that the model itself amounts to two coupled real equations as the Brusselator. This implies that atomic variables must not appear in the model; this can be obtained by adiabatically eliminating the atomic variables or by directly introducing a nonlinear term (expressed in terms of the field envelope) in the field envelope equation.

In the formulation of the LLE, the choice of the nonlinearity was dictated by the criterion of maximum simplicity. Quadratic nonlinearities are not appropriate because they involve two envelopes, one for the fundamental frequency and one for the second harmonic. Therefore the simplest choice is that of a cubic nonlinearity, i.e. the Kerr nonlinearity. As a conclusion, the LLE involves the following terms: the time derivative, the transverse Laplacian which describes diffraction, the Kerr nonlinear term, a term which describes the driving input field and two terms related to the ring cavity

$$
\frac{\partial E}{\partial \bar{t}}=E_{I}-E-i \theta E+i \eta|E|^{2} E+i \nabla_{\perp}^{2} E
$$

with

$$
\nabla_{\perp}^{2} E=\frac{\partial^{2} E}{\partial \bar{x}^{2}}+\frac{\partial^{2} E}{\partial \bar{y}^{2}} .
$$

In equation (4) $E$ and $E_{I}$ (the input field amplitude) are appropriately normalized in order to reduce to a minimum the number of parameters which appear in the equation (see [1] and Sect. 27.1 in Ref. [21]), the normalization involves also the nonlinear susceptibility $\left.\chi^{(3)}\right)$. The 
quantities $\bar{t}, \bar{x}, \bar{y}$ are normalized coordinates defined as

$$
\bar{t}=\kappa t=\frac{\tilde{c} T}{\mathcal{L}} t, \bar{x}=\frac{x}{x_{T}}, \bar{y}=\frac{y}{x_{T}}, \quad \text { with } \quad x_{T} \propto \frac{\sqrt{\lambda \mathcal{L}}}{T},
$$

where $\kappa$ is the cavity decay rate, $x_{T}$ is the characteristic scale of transverse optical patterns and $\lambda$ is the wavelength. The first term on the r.h.s. of equation (4) introduces the input field, which is assumed independent of the spatial variables and is usually assumed independent of $t$, but may also be time-dependent when a pulse is injected in the cavity in addition to the stationary field. The second term describes the escape of photons from the cavity, the third is the detuning term, where $\theta$ is defined in equation (3). In the nonlinear term, the parameter $\eta$ is equal to +1 in the self-focussing case, to -1 in the self-defocussing case. $\nabla_{\perp}^{2}$ is the transverse Laplacian.

At this point two remarks are in order. First, in the low transmission limit in which the LLE is valid, the "map" procedure to calculate the time evolution roundtrip after roundtrip [41,42] is inconvenient because the roundtrip cavity time is not the correct time scale, and this method requires an exceedingly large number of iterations to converge.

The second remark is that a realistic model for nonlinear chemical reactions requires many more than two differential equations, as described in [43]. On the other hand, the LLE is a realistic model which, despite its relative simplicity, captures the essential physical elements of the system it describes and is capable of governing a complex multimodal reality, a large variety of pattern formation phenomena not only transverse as in the case of equation (4) but also longitudinal as in the case of the following equation (13), and in the frequency domain.

If we set $\theta=\eta \bar{\theta}$ the LLE (4) becomes

$$
\frac{\partial E}{\partial \bar{t}}=E_{I}-E-i \eta\left(\bar{\theta}-|E|^{2}\right) E+i \nabla_{\perp}^{2} E .
$$

If we define

$$
X=|E|^{2}, \quad Y=E_{I}^{2}
$$

where $E_{I}$ is assumed real, the homogeneous $\left(\nabla_{\perp}^{2} E=0\right)$, stationary $(\partial E / \partial \bar{t}=0)$ solutions obey the cubic equation

$$
Y=X\left[1+(\bar{\theta}-X)^{2}\right]
$$

that was formulated in the paper [6] which reported on the first experimental observation of optical bistability. As a matter of fact, as it is well known for $\theta>\sqrt{3}$ the stationary curve (9) of $X$ as a function of $Y$ is $S$-shaped, and the negative-slope segment of the steady-state curve is unstable (see Fig. 11.6 of Ref. [21]).

The linear stability analysis of $[1,44]$ showed that under appropriate conditions one or more segments of the homogeneous stationary curve with positive slope become unstable (modulational instability), so that there is the possibility of the formation of a stable stationary pattern. The calculation of the modulated solution was done analytically in $[1,44]$ in the case of one transverse dimension, and the result was that the bifurcation is supercritical

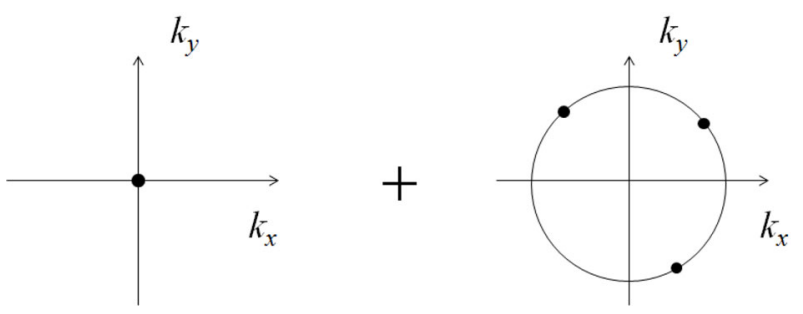

Fig. 3. The lefthand figure indicates that the (unstable) stationary state corresponds to the origin of the Fourier plane (far field). The righthand figure indicates the points in the Fourier plane corresponding to the tilted plane waves emitted just beyond the spatial instability threshold.

(a)

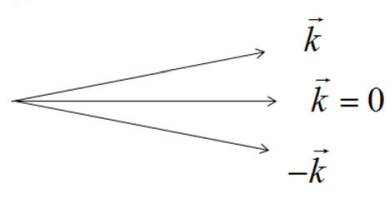

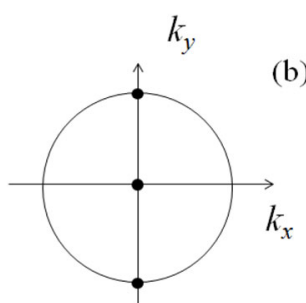

Fig. 4. (a) In the four-wave mixing process, two symmetrically tilted plane waves may be emitted just beyond the instability threshold; (b) Fourier plane configuration of the field described by (a).

(and therefore the modulated solution is stable near the bifurcation point) when $\bar{\theta}>41 / 30$, and has a typical sinusoidal configuration near the instability threshold.

In [45] Grynberg showed that nonlinear optics provides a simple guideline to predict which kind of patterns arise from a spatial modulational instability associated with a certain optical nonlinearity. The field configuration beyond the instability threshold can be written in the form

$$
E(x, y)=E_{s t} \mathrm{e}^{i \mathbf{0} \cdot \mathbf{x}}+\sum_{j} b_{j} \mathrm{e}^{i \mathbf{k}_{j} \cdot \mathbf{x}},
$$

where $E_{s t}$ is the value of $E$ in the unstable stationary state which is considered, $\mathbf{x}=(x, y)$ is the position vector in the transverse plane and $\mathbf{k}=\left(k_{x}, k_{y}\right)$ is the transverse wave vector. In the Fourier plane of the variables $k_{x}, k_{y}$, i.e. in the far field, equation (10) corresponds to what shown in Figure 3. The exponential factor in the first term in the r.h.s. of equation (10), which is equal to unity, has been introduced to indicate that this term corresponds to the point $\mathbf{k}=\mathbf{0}$ in the Fourier plane. The vectors $\mathbf{k}_{j}$ lie on the critical circle which is associated with the instability (see Sect. 27.2 of Ref. [21]).

The Kerr nonlinearity corresponds to the process of four-wave mixing. A possibility is that two pump photons which propagate in the longitudinal direction $z$ are absorbed by the medium, and that simultaneously two photons which propagate symmetrically (transverse wave vectors $\mathbf{k},-\mathbf{k}$ ) are emitted (Fig. 4a). This kind of process leads to a far field with a central spot corresponding to the pump wave plus two symmetrical spots corresponding to the two tilted waves (Fig. 4b). Expressing in formulas, 


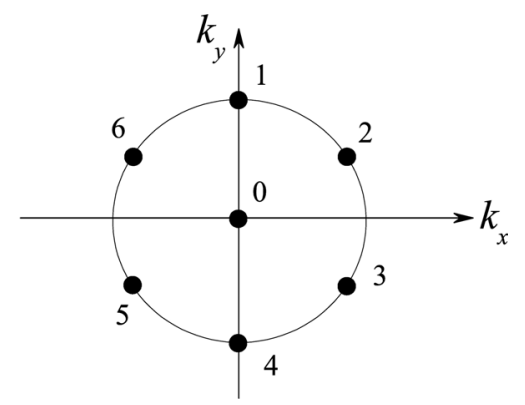

Fig. 5. The generation of a hexagonal far field (see text). Reprinted figure from reference [19], with permission from Cambridge University Press.

this amounts to

$$
\begin{aligned}
E(\mathbf{x}) & =E_{s t} \mathrm{e}^{i \mathbf{0} \cdot \mathbf{x}}+\sigma \mathrm{e}^{i \phi_{+}} \mathrm{e}^{i \mathbf{k} \cdot \mathbf{x}}+\sigma \mathrm{e}^{i \phi_{-}} \mathrm{e}^{-i \mathbf{k} \cdot \mathbf{x}} \\
& =E_{s t}+2 \sigma \cos \left(\mathbf{k} \cdot \mathbf{x}+\frac{\phi_{+}-\phi_{-}}{2}\right) \mathrm{e}^{i \frac{\phi_{+}+\phi_{-}}{2}} .
\end{aligned}
$$

Due to the rotational symmetry any rotated version of Figure $4 \mathrm{~b}$ is possible.

Let us now consider for a while the case of one transverse dimension which can be realized, for example, in a waveguide configuration. In this case equation (11) reduces to

$$
E(y)=E_{s t}+2 \sigma \cos \left(k y+\frac{\phi_{+}-\phi_{-}}{2}\right) \mathrm{e}^{i \frac{\phi_{+}+\phi_{-}}{2}} .
$$

A remark of paramount importance is now that the two photons, emitted in symmetrically tilted directions, are in a state of quantum entanglement (they are precisely correlated, for example, in energy and momentum). This fact is fundamental for the quantum aspects of optical patterns. For instance, the difference between the intensities of the two symmetrically tilted beams is squeezed, i.e. exhibits fluctuations below the shot noise level [46]. In turn, such quantum aspects are basic for the field of quantum imaging $[47,48]$.

Let us now turn the case of two transverse dimensions, in which equation (11) corresponds to a roll (i.e. stripe) pattern. However, as shown in [45], in 2D the stripe pattern created by the FWM process is unstable (Fig. 5). As a matter of fact, a second FWM process creates two photons (2 and 6 ) from 0 and 1 , and the pair 3 and 5 from 0 and 4 , all with conservation of the total transverse photon momentum, and this gives rise to a hexagonal structure in the far field. Gomila and Colet $[49,50]$ analyzed the complex scenario of hexagonal patterns which arise in the near field over the parameter space, in many cases the pattern exhibits a dynamical (and in some cases chaotic) behavior.

\subsection{Spatial cavity solitons}

In the field of spatial pattern formation one meets, in addition to global patterns the elements of which are mutually well correlated, also the case of localized structures formed

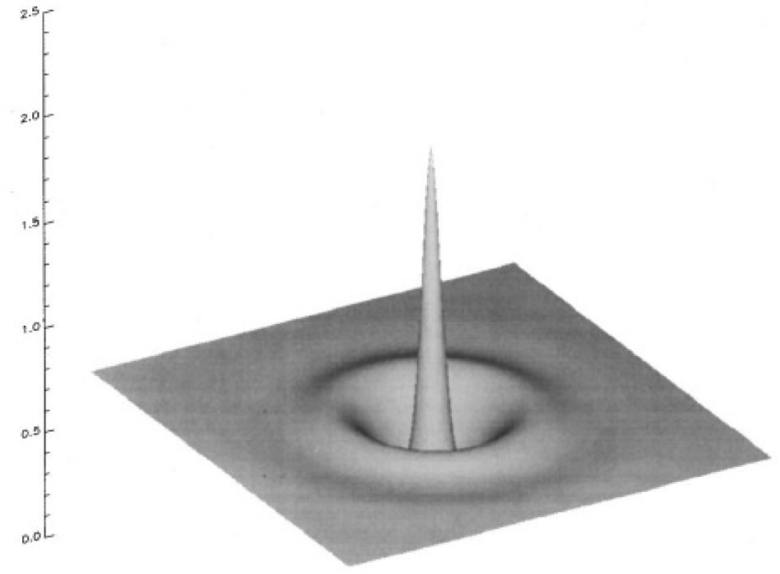

Fig. 6. A typical Kerr cavity soliton, showing a bright peak on a darker homogeneous background with a few weak diffraction rings. The modulus of the normalized intracavity field is plotted as a function of the transverse coordinates $x$ and $y$. Reprinted figure from reference [50] with permission from the Optical Society of America.

by one or more elements that are independent provided that they are not too close to one another (see e.g. [51]). In the framework of nonlinear optics, the possibility of localized structures was first predicted by Tlidi, Mandel and Lefever [52]; they are usually called with the name of cavity solitons introduced by Firth and correspond to isolated intensity peaks.

Cavity solitons in the framework of the LLE were analyzed over the parameter space by Firth et al. [53] (see Fig. 6). Their theoretical investigation showed also that, when the driving field intensity is increased, the cavity solitons may start breathing, i.e. their height and width oscillate periodically in time.

Reviews of the topic of cavity solitons can be found in $[54,55]$ and in chapter 30 of reference [21]. Figure 7 illustrates the standard procedure used to generate cavity solitons by means of optical resonators containing nonlinear materials. The energy is provided to the system by a broad area, coherent and stationary holding beam that is injected into the cavity. The system lies initially in a uniform stationary state. In order to create a cavity soliton, one injects into the cavity a short and narrow "writing" pulse. Provided the pulse is (approximately) in phase with the holding beam, the intensity locally increases and, in the output transverse profile, one has the formation of a bright intensity peak. When the writing pulse goes out of the cavity, the peak persists where it has been excited. Therefore the cavity soliton remains in the memory of the system. By injecting other writing pulses in different locations of the transverse section one can turn on as many cavity solitons as one likes, provided that the distances among them are larger than a minimal distance below which they interact. In order to switch a cavity soliton off, with no consequences for the other cavity solitons, it suffices to shoot, at the location where a cavity soliton lies, an "erasing" pulse similar to the "writing" one but 


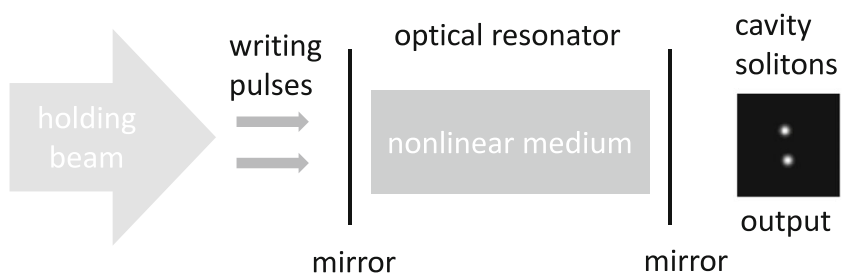

Fig. 7. A coherent, stationary, quasi plane-wave holding beam drives the optical cavity containing a nonlinear medium. The injection of narrow laser pulses creates persistent localized intensity peaks in the output (cavity solitons). Reprinted figure from reference [21] with permission from Cambridge University Press.

with (approximately) opposite phase with respect to the holding beam.

A fundamental property of cavity solitons is that they move spontaneously when they are in presence of phase or amplitude gradients in the holding beam, or a temperature gradient in the material. For example, in a phase gradient a cavity soliton moves spontaneously towards the nearest local maximum of the phase profile, and remains there indefinitely. By exploiting this mechanism it is possible to introduce appropriate phase modulations in the holding beam and realize reconfigurable arrays of cavity solitons, serial-to-parallel converters etc.

Because of its paradigmatic simplicity, the LLE has been extensively used by the optical community, and it has been even called the "hydrogen atom" of nonlinear cavities [56]. However, only recently Kerr cavities with a large aspect ratio have been realized, and transverse patterns and solitons observed [57,58]. For this reason cavity solitons have been analyzed theoretically mainly in a model which describes a semiconductor microresonator (see Sect. 30.3 of Ref. [21]). This model is substantially more complex than the LLE because it includes, in addition to the time evolution equation for the field envelope, also a time evolution equation for a variable which is immediately linked to the carrier density in the semiconductor. Another important difference is that this system is active, i.e. it has population inversion, even it works as an amplifier because it is kept slightly below the threshold for laser emission.

Such a system has been realized experimentally using broad area (circular section with diameter of $150 / 200 \mu \mathrm{m}$ ) VCSELs below threshold, in a configuration which satisfies very well all the conditions of the low transmission and of the singlemode limit. This has led to the first experimental observation of cavity solitons [59] with their writing and erasing and, subsequently, of arrays of cavity solitons [60].

\subsection{The temporal/longitudinal version of the LLE}

In formulating the temporal/longitudinal version of the LLE the authors of [7] were inspired by the analogy between two kinds of Hamiltonian solitons

- temporal solitons, which propagate without deformation in the longitudinal direction $z$ and are governed by a nonlinear Schroedinger equation with a second derivative with respect to the retarded time, which describes group velocity dispersion;

- spatial solitons, which are "tubes" of radiation described by a similar Schroedinger equation, with dispersion replaced by diffraction, i.e. with the transverse Laplacian;

and they extended this analogy to the dissipative case of cavity solitons, proceeding in reverse order with respect to the Hamiltonian configuration.

They considered [7] a nonlinear fiber loop with an input/output mirror, in the practical realizations the mirror is replaced by input and output fiber couplers They started from the nonlinear Schroedinger equation with dispersion, combining it with the boundary condition of the cavity. Using the low transmission limit but not the singlemode limit, after a long sequence of steps one arrives at the temporal/longitudinal version of the LLE

$$
\frac{\partial E}{\partial \bar{t}}=E_{I}-E-i \theta E+i|E|^{2} E-i \bar{\eta} \frac{\partial^{2} E}{\partial \bar{\tau}^{2}}
$$

where $\bar{t}$ is defined by equation (6), $\bar{\tau}$ is also dimensionless and proportional to the retarded time $\tau=t-z / v_{g}, v_{g}$ being the group velocity of light, $\bar{\eta}$ is equal to +1 in the case of normal dispersion and to $(-1)$ in the case of anomalous dispersion. As in the case of the spatial LLE (4), E and $E_{I}$ are normalized in such a way that the number of parameters is reduced to the minimum. It is evident that, apart from the presence of the parameters $\eta$ and $\bar{\eta}$, the temporal/longitudinal version (13) corresponds to the transverse version (4) with the diffraction term replaced by the group velocity dispersion term.

While the transverse model involves the temporal variable $\bar{t}$ and the two spatial variables $x, y$, the temporal/ longitudinal model involves two temporal variables. The first one is the same slow variable $\bar{t}$ as in the transverse version, which describes phenomena occurring on the long scale of the cavity decay time, the second one is the fast temporal variable $\bar{\tau}$, which describes phenomena occurring on the short scale of the cavity roundtrip time. Therefore the temporal/longitudinal version of the LLE is formally identical to the transverse version in $1 \mathrm{D}$.

The dependence on the retarded time corresponds to a $1 \mathrm{D}$ pattern in the longitudinal direction $z$, and the pattern circulates in the ring fiber loop with the velocity of light.

More precisely, in the case of anomalous dispersion $\bar{\eta}=$ -1 the temporal/longitudinal equation (13) is formally identical to the transverse equation (4) in 1D in the selffocussing case $\eta=1$. In the case of normal dispersion $\bar{\eta}=1$, the complex conjugate of the temporal/longitudinal version equation (13) reads

$$
\frac{\partial E^{*}}{\partial \bar{t}}=E_{I}-E^{*}-i\left(\left|E^{*}\right|^{2}-\theta\right) E^{*}+i \frac{\partial^{2} E^{*}}{\partial \bar{\tau}^{2}},
$$

where, as before, we have assumed that $E_{I}$ is real. Equation (14) is formally identical to the transverse $1 \mathrm{D}$ version of equation (7) in the self-defocussing case $\eta=-1$, provided that $E$ is replaced by $E^{*}$ and $\bar{\theta}$ is replaced by $\theta$. The replacement of $E$ by $E^{*}$ is immaterial for the intensity. 


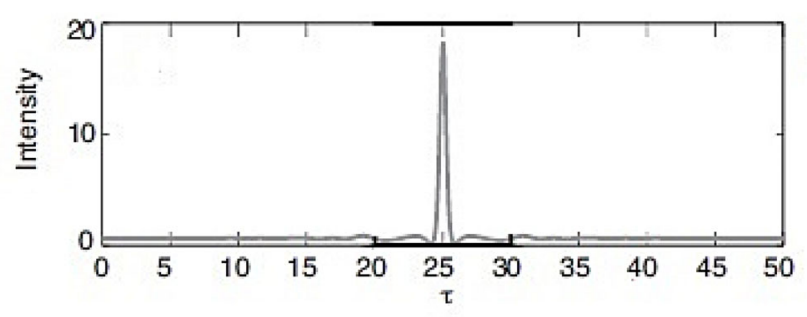

Fig. 8. Intensity profile of a temporal cavity soliton. Reprinted figure from reference [62] with permission from Optical Society of America.

A key point is that the temporal/longitudinal version of the LLE can be easily realized experimentally, because standard silica fibers display a perfect Kerr nonlinearity and the ring cavity can be easily constructed using off the shelf optical components and fibers.

The results that we have described before for the 1D case of the transverse LLE hold unaltered for the tempo$\mathrm{ral} /$ longitudinal version, provided the transverse variable $y$ is replaced by the retarded time $\tau$ and the spatial frequency $k$ is replaced by the temporal frequency offset $\Omega$. In particular this is true for the modulational instability and for the pattern (12) which arises near the instability threshold. Such sinusoidal patterns have been observed experimentally by Coen and Haelterman [61,62].

A temporal cavity soliton is a narrow pulse which circulates indefinitely (with the velocity of light) in the fiber cavity without deformation, apart from fluctuations, with a period equal to the cavity roundtrip time (Fig. 8). In the case of the transverse LLE, cavity solitons sit on the pedestal of a stable homogeneous stationary solution, in the temporal/longitudinal version they sit on the pedestal of a stable stationary solution (stationary with respect to both $t$ and $\tau$ ). Temporal cavity solitons are excited by injecting into the cavity short address pulses that add to the stationary driving field.

It is interesting to note [26] that for $\theta>0$ the function

$$
E_{c s}(\bar{\tau})=\sqrt{2 \theta} \operatorname{sech}(\sqrt{\theta} \bar{\tau})
$$

sent into the cavity just once. Following this procedure the authors of [63] have been able to store the acronym ULB of Universitè Libre Bruxelles into a sequence of 15 bits, and the fiber cavity operates as an all-optical memory [64]. It is claimed that, using appropriate techniques, there is a potential of 45 kbits memory at $25 \mathrm{Gbits} / \mathrm{s}$. A later experiment [65] reported on the observation of breathing cavity solitons which oscillate periodically over the slow time scale $t$.

Recent experiments have shown that by introducing appropriate phase modulations in the driving field it is possible to

- write and erase temporal cavity solitons at desired temporal locations [66];

- operate a "temporal tweezing" of light through the trapping and manipulation of temporal cavity solitons [67].

Pattern formation in fiber ring cavities is analyzed also in reference [68].

It is interesting to observe that 3D pattern formation, i.e. simultaneously in the longitudinal and in the transverse directions, in the framework of the LLE has been studied theoretically in [69]. On the other hand 3D cavity solitons are not possible in the LLE [70].

\subsection{Broadband Kerr frequency combs}

The microresonators which have demonstrated Kerr frequency combs (see e.g. [12-17,22-25]) realize ideally the assumptions on which the LLE is based, especially the Kerr nonlinearity and the high- $Q$ condition, reaching $Q$ values on the order of $10^{6}$ or even $10^{9}$ or more [30]. A main advantage of the high- $Q$ condition is that it allows to obtain important nonlinear effects even with a weak nonlinearity, in accord with the low transmission limit.

The technological progress in the field of photonics achieved from the time of reference [1] to nowadays has been spectacular, and the pattern formation in the longitudinal direction of ring cavities, associated with the experimental observation of broadband frequency combs, occurs in microcavities with a length on the order of $10 \mathrm{~mm}$ or less, a drastic difference from the long cavity of [37]. Such Kerr microcavities are operated with driving frequencies convenient for telecommunication, can be embedded on chip, can be integrated in fiber networks and are compatible with CMOS/metal oxide semiconductors. Such properties make this approach quite promising for applications. In optical coherent telecommunications one can use each element of the comb to transmit data [28,29]. Other examples of possible fields of application are ultrastable microwave generation, spectroscopy with mid-IR combs, quantum technologies [30], and this scenario motivates the noteworthy worldwide effort which supports such an approach.

Since Kerr microcavities are operated as a passive system without population inversion, they can represent a system which is less noisy then, for example, a modelocked laser, a feature which can be beneficial for the 
stability of the combs. Very important in this connection is the fact that the frequencies of the comb are robustly phase locked [71], a property that arises spontaneously together with the instability that creates the spatial pattern and the frequency comb.

In the case of frequency combs associated with a cavity soliton, the frequency spacing between adjacent elements of the comb is equal to the free spectral range of the cavity; for combs associated with Turing patterns the frequency spacing is a multiple of the free spectral range [26,30].

Noteworthy is also the recent progress in the field of quantum effects in frequency combs, theoretical [72] and experimental. In the paper [73] Lipson, Gaeta and collaborators report on the first experimental observation of "on-chip squeezing", i.e. of sub-shot noise fluctuations in the intensity difference between two modes of the comb symmetrically positioned with respect to the central mode corresponding to the laser frequency which is injected into the cavity. This effect closely corresponds to that theoretically predicted in $[32,46]$ (see also [72]). Therefore the results of [73] represent the first experimental observation of a quantum effect associated with a spatial pattern in a microcavity (and in frequency combs as well).

\section{Derivation of the temporal/longitudinal LLE from the Maxwell-Bloch equations}

In the low transmission limit, the Maxwell-Bloch equations read (see Chap. 11 in Ref. [21])

$$
\begin{aligned}
\frac{\partial F}{\partial t}+\tilde{c} \frac{\partial F}{\partial z} & =-\kappa[(1+i \theta) F-y+2 C P] \\
\frac{\partial P}{\partial t} & =-\gamma_{\perp}[(1+i \Delta) P-F D] \\
\frac{\partial D}{\partial t} & =-\gamma_{\|}\left[\frac{1}{2}\left(F P^{*}+F^{*} P\right)+D-1\right]
\end{aligned}
$$

where $F, y, P$ and $D$ are proportional to the field envelope $E$ of equation (4), to the input field amplitude $E_{I}$, to the atomic polarization and to the population difference, respectively (see Sect. 4.3 and Eq. (8.15) in Ref. [21]). $\gamma_{\perp}$ and $\gamma_{\|}$are the transverse and longitudinal atomic relaxation rates, respectively. The atomic detuning parameter is defined as $\Delta=\left(\omega_{a}-\omega_{0}\right) / \gamma_{\perp}$, with $\omega_{a}$ being the atomic Bohr transition frequency of the two-level atoms. Note that in reference [31] the atomic detuning $\Delta$ is defined with reverse sign.

Again, the length of the atomic sample is assumed equal to the cavity length. The symbol $\tilde{c}$ is defined as $\tilde{c}=c / n_{B}$, where $c$ is the light velocity in vacuum and $n_{B}$ accounts for the possible presence of a background medium different from the two-level atoms described by the variables $P$ and $D$.

We will derive the temporal/longitudinal LLE from equation (16) following two different paths. One is more heuristic and direct, and more in line with common procedures used in nonlinear optics; it is described in Appendix A. The other one is more rigorous because it takes into account precisely the order of magnitude of the quantities in play, which must be assumed to arrive at the LLE; it is described in this section.

Equation (16) admit the homogeneous stationary solution

$y^{2}=x^{2}\left[\left(1+\frac{2 C}{1+\Delta^{2}+x^{2}}\right)^{2}+\left(\theta-\frac{2 C \Delta}{1+\Delta^{2}+x^{2}}\right)^{2}\right]$,

where $x=|F|$, which is the well-known input-output relation for optical bistability [21]. Let us consider the dispersive limit of such an equation, heuristically defined as the limit in which the frequency of the input field is so far from the atomic resonance frequency that $|\Delta| \gg 1$ and $x^{2} / \Delta^{2} \ll 1$. In that limit the stationary equation can be approximated as

$y^{2}=x^{2}\left[\left(1+\frac{2 C}{\Delta^{2}}-\frac{2 C x^{2}}{\Delta^{4}}\right)^{2}+\left(\theta-\frac{2 C}{\Delta}+\frac{2 C x^{2}}{\Delta^{3}}\right)^{2}\right]$.

We can now define more precisely the dispersive limit through a smallness parameter $\epsilon$ such that [32]

$$
\begin{array}{cc}
\Delta=O\left(\epsilon^{-3}\right), & x, y=O\left(\epsilon^{-2}\right), \\
2 C=O\left(\epsilon^{-5}\right), & \theta=\theta_{0}+\frac{2 C}{\Delta},
\end{array}
$$

with $\theta_{0}=O(1), \theta=O\left(\epsilon^{-2}\right)$ and we define the scaled quantities

$$
\tilde{y}=\sqrt{\frac{2 C}{|\Delta|^{3}}} y=O(1), \quad \tilde{x}=\sqrt{\frac{2 C}{|\Delta|^{3}}} x=O(1)
$$

and the parameter

$$
\eta=-\frac{|\Delta|}{\Delta}
$$

It turns out that the stationary equation at order 0 in $\epsilon$ is

$$
\tilde{y}^{2}=\tilde{x}^{2}\left[1+\left(\theta_{0}-\eta \tilde{x}^{2}\right)^{2}\right]
$$

which coincides with equation (9) if we set $\theta_{0}=\eta \bar{\theta}, X=$ $\tilde{x}^{2}$, and $Y=\tilde{y}^{2}$. On the basis of these considerations we rewrite equation (16) in terms of the new variables ${ }^{1}$

$$
\tilde{F}=\sqrt{\frac{2 C}{|\Delta|^{3}}} F, \quad \tilde{P}=\sqrt{\frac{2 C}{|\Delta|^{3}}} \Delta P,
$$

${ }^{1}$ In reference [21], Section 13.3, the normalization factor is $\sqrt{|\theta| / \Delta^{2}}$, instead of $\sqrt{2 C /|\Delta|^{3}}$ which appears in equation (23), but it is easy to check that they basically coincide because, in the limit (19), $2 C$ can be replaced by $\Delta \theta=|\Delta \theta|$ because $2 C>0$, hence $\sqrt{2 C /|\Delta|^{3}} \simeq \sqrt{|\theta| / \Delta^{2}}$. 
1 and of the parameters $\theta_{0}, \tilde{y}$, and $\eta$ as

$$
\begin{aligned}
\frac{\partial \tilde{F}}{\partial t}+\tilde{c} \frac{\partial \tilde{F}}{\partial z} & =-\kappa\left[\left(1+i \theta_{0}\right) \tilde{F}-\tilde{y}+\frac{2 C}{\Delta}(\tilde{P}+i \tilde{F})\right], \\
\frac{\partial \tilde{P}}{\partial t} & =-\gamma_{\perp}[(1+i \Delta) \tilde{P}-\Delta \tilde{F} D], \\
\frac{\partial D}{\partial t} & =-\gamma_{\|}\left[-\frac{\eta}{2} \frac{\Delta^{2}}{2 C}\left(\tilde{F} \tilde{P}^{*}+\tilde{F}^{*} \tilde{P}\right)+D-1\right] .
\end{aligned}
$$

The quantities $\tilde{F}, \tilde{P}$ and $D$ obey the periodic boundary condition $\tilde{F}(z=0, t)=\tilde{F}(z=\mathcal{L}, t)$ etc. Hence we can introduce the modal expansion $[21,32]$

$$
\left\{\begin{array}{l}
\tilde{F}(z, t) \\
\tilde{P}(z, t) \\
D(z, t)
\end{array}\right\}=\sum_{n}\left\{\begin{array}{l}
f_{n}(t) \\
p_{n}(t) \\
d_{n}(t)
\end{array}\right\} \mathrm{e}^{-i n \alpha(t-z / \tilde{c})}
$$

where the index $n$ runs over the values $n=0, \pm 1, \pm 2, \ldots$, $d_{-n}^{*}=d_{n}$, and $\alpha=2 \pi \tilde{c} / \mathcal{L}$ is the free spectral range. Introducing this expansion into equations (24), we obtain the following system of coupled equations

$$
\begin{aligned}
\frac{d f_{n}}{d t}= & -\kappa\left[\left(1+i \theta_{0}\right) f_{n}-\tilde{y} \delta_{n, 0}+\frac{2 C}{\Delta}\left(p_{n}+i f_{n}\right)\right] \\
\frac{d p_{n}}{d t}= & -\gamma_{\perp}\left[\left(1+i \Delta_{n}\right) p_{n}-\Delta \sum_{n^{\prime}} f_{n-n^{\prime}} d_{n^{\prime}}\right], \\
\frac{d d_{n}}{d t}= & \gamma_{\|}\left[\frac{\eta}{2} \frac{\Delta^{2}}{2 C} \sum_{n^{\prime}}\left(f_{-n^{\prime}}^{*} p_{n-n^{\prime}}+f_{n^{\prime}} p_{n^{\prime}-n}^{*}\right)+\delta_{n, 0}\right] \\
& -d_{n}\left(\gamma_{\|}-i n \alpha\right),
\end{aligned}
$$

where we have introduced the atomic detuning at the frequency $\omega_{0}+n \alpha$

$$
\Delta_{n}=\Delta-n \frac{\alpha}{\gamma_{\perp}}=\frac{\omega_{a}-\left(\omega_{0}+n \alpha\right)}{\gamma_{\perp}} .
$$

The stationary solutions, obtained by setting $d f_{n} / d t=$ $d p_{n} / d t=d d_{n} / d t=0$ are singlemode, i.e. only the mode $n=0$ contributes.

If equations (25) are linearized around an exact stationary solution one obtains the linearized equations that govern the multimode instability of optical bistability studied in [8,9] and in Sections 24.1.1 and 24.1.2 of [21].

We complete the definition of the dispersive limit by assuming

$$
\frac{|n| \alpha}{\gamma_{\perp}}, \frac{|n| \alpha}{\gamma_{\|}}=O\left(\epsilon^{-2}\right) .
$$

This allows to determine which are the effective variation rates of the dynamical variables. In the equation for the $f_{n}$ 's all the terms in the square bracket are of order 1 but the large coefficient $2 C / \Delta=O\left(\epsilon^{-2}\right)$ which, however, multiplies $\left(p_{n}+i f_{n}\right)$. Since we shall show that in the dispersive limit $p_{n}=-i f_{n}+O(\epsilon)$, the temporal variation rate for the $f_{n}$ 's is $O\left(\kappa \epsilon^{-1}\right)$.

On the other hand, the actual variation rates for the variables $p_{n}$ 's and $d_{n}$ 's are, respectively, $\gamma_{\perp}|\Delta|=$ $O\left(\gamma_{\perp} \epsilon^{-3}\right)$ for the $p_{n}$ 's, and $\gamma_{\|} \Delta^{2} /(2 C)=O\left(\gamma_{\|} \epsilon^{-1}\right)$ for $d_{0}, n \alpha \gamma_{\|}=O\left(\gamma_{\|} \epsilon^{-2}\right)$ for $d_{n \neq 0}$. Since we have assumed in equation (27) that $\gamma_{\perp}$ and $\gamma_{\|}$have the same magnitude, an adiabatic elimination of the atomic variables is justified if $\kappa / \gamma_{\|} \approx \kappa / \gamma_{\perp}=O(\epsilon)$ or smaller.

By imposing zero time derivatives at the 1.h.s. of equations (25b) and (25c) we obtain

$$
\begin{aligned}
p_{n}= & \frac{\Delta}{1+i \Delta_{n}} \sum_{m} f_{n-m} d_{m} \\
d_{n}\left(1-i n \frac{\alpha}{\gamma_{\|}}\right)= & \frac{\eta}{2} \frac{\Delta^{2}}{2 C} \sum_{l}\left(f_{-l}^{*} p_{n-l}+f_{l} p_{l-n}^{*}\right) \\
& +\delta_{n, 0},
\end{aligned}
$$

and, by inserting equation (28) in equation (29), we can write

$$
\begin{aligned}
d_{n}\left(1-i n \frac{\alpha}{\gamma_{\|}}\right)= & \frac{\eta}{2} \frac{\Delta^{3}}{2 C} \sum_{l, m}\left(\frac{f_{-l}^{*} f_{n-l-m} d_{m}}{1+i \Delta_{n-l}}\right. \\
& \left.+\frac{f_{l} f_{l-n-m}^{*} d_{-m}}{1-i \Delta_{l-n}}\right)+\delta_{n, 0} .
\end{aligned}
$$

Let us now consider the double sum at the r.h.s of this equation. It can be rewritten as

$$
\begin{aligned}
& \sum_{l, m}\left(\frac{f_{-l}^{*} f_{n-l-m} d_{m}}{1+i \Delta_{n-l}}+\frac{f_{l} f_{l-n+m}^{*} d_{m}}{1-i \Delta_{l-n}}\right) \\
& =\sum_{m, j}\left(\frac{f_{m+j}^{*} f_{n+j} d_{m}}{1+i \Delta_{n+m+j}}+\frac{f_{n+j} f_{m+j}^{*} d_{m}}{1-i \Delta_{j}}\right) \\
& =\sum_{m, j} f_{m+j}^{*} f_{n+j} d_{m} \frac{2-i(n+m) \alpha / \gamma_{\perp}}{\left(1+i \Delta_{n+m+j}\right)\left(1-i \Delta_{j}\right)} \\
& \approx \frac{2}{\Delta^{2}} \sum_{m, j} f_{m+j}^{*} f_{n+j} d_{m}\left[1-i(n+m) \frac{\alpha}{2 \gamma_{\perp}}\right],
\end{aligned}
$$

where in the first line we have replaced $m$ with $-m$ in the second sum, in the second line we have replaced $l$ with $-m-j$ in the first sum and with $n+j$ in the second sum, in the third line we have used the definition (26) of $\Delta_{n}$, and in the last line we have approximated $\Delta_{n+m+j}$ and $\Delta_{j}$ with $\Delta$. By replacing equation (31) in equation (30) we obtain

$$
\begin{aligned}
& d_{n}\left(1-i n \frac{\alpha}{\gamma_{\|}}\right)=\delta_{n, 0} \\
& \quad+\eta \frac{\Delta}{2 C} \sum_{m, j} f_{m+j}^{*} f_{n+j} d_{m}\left[1-i(n+m) \frac{\alpha}{2 \gamma_{\perp}}\right] .
\end{aligned}
$$

For $n=0$ the leading terms of this equation are $d_{0}$ and $\delta_{n .0}$ which is of order $\epsilon^{0}$, while the nonlinear term is $O\left(\epsilon^{2}\right)$. For $n \neq 0$ the leading term is the second term on the lefthand 

we look for solutions up to order $\epsilon^{2}$

$$
d_{n}=d_{0}^{(0)} \delta_{n, 0}+\frac{\Delta}{2 C} d_{n}^{(2)}+O\left(\epsilon^{4}\right),
$$

$$
\begin{aligned}
d_{0}^{(0)} \delta_{n, 0} & +\left(1-i \frac{n \alpha}{\gamma_{\|}}\right) \frac{\Delta}{2 C} d_{n}^{(2)} \\
& =\delta_{n, 0}+\eta \frac{\Delta}{2 C} \sum_{j} f_{j}^{*} f_{n+j} d_{0}^{(0)}\left(1-i \frac{n \alpha}{2 \gamma_{\perp}}\right) .
\end{aligned}
$$

6 For $n=0$ we get

$$
d_{0}^{(0)}=1, \quad d_{0}^{(2)}=\eta \sum_{j}\left|f_{j}\right|^{2},
$$

7 and for $n \neq 0$

$$
d_{n}^{(2)}=\eta \frac{\gamma_{\|}}{2 \gamma_{\perp}} \sum_{j} f_{j}^{*} f_{n+j} .
$$

The two expressions for the second order corrections have the same form if we assume the non-radiative limit $\gamma_{\|}=$ $2 \gamma_{\perp}$. We note incidentally that this condition on $\gamma_{\|}$and $\gamma_{\perp}$ is the most convenient for squeezing [74]. With this assumption we can write

$$
d_{n}=\delta_{n, 0}+\eta \frac{\Delta}{2 C} \sum_{j} f_{j}^{*} f_{n+j},
$$

and inserting this expression in equation (28) we find an expression of the $p_{n}$ 's in terms only of the $f_{n}$ 's

$$
p_{n}=\frac{\Delta}{1+i \Delta_{n}}\left[f_{n}+\eta \frac{\Delta}{2 C} \sum_{m, j} f_{n-m} f_{j}^{*} f_{m+j}\right] .
$$

We want to evaluate this quantity consistently up to order $O\left(\epsilon^{2}\right)$ since in equation (25a) $p_{n}$ is multiplied by $2 C / \Delta=$ $O\left(\epsilon^{-2}\right)$. To this aim we expand the pre-factor in the linear term of equation (38) as

$$
\begin{aligned}
\frac{\Delta}{1+i \Delta_{n}} & \approx \frac{\Delta}{i \Delta_{n}}=-\frac{i}{1-n \frac{\alpha}{\gamma_{\perp} \Delta}} \\
& \approx-i\left(1+\frac{n \alpha}{\gamma_{\perp} \Delta}+\frac{n^{2} \alpha^{2}}{\gamma_{\perp}^{2} \Delta^{2}}\right),
\end{aligned}
$$

while in the nonlinear term, which is $O\left(\epsilon^{2}\right)$, we keep only the dominant term $-i$. Therefore

$$
\begin{aligned}
p_{n}= & -i f_{n}-i \frac{n \alpha}{\gamma_{\perp} \Delta} f_{n}-i \frac{n^{2} \alpha^{2}}{\gamma_{\perp}^{2} \Delta^{2}} f_{n} \\
& -i \eta \frac{\Delta}{2 C} \sum_{m, j} f_{n-m} f_{j}^{*} f_{m+j}+O\left(\epsilon^{3}\right)
\end{aligned}
$$

and the modal equations (25a) reduce to

$$
\begin{aligned}
\frac{d f_{n}}{d t}= & -\kappa\left[\left(1+i \theta_{0}-i \frac{2 C}{\Delta} \frac{n \alpha}{\gamma_{\perp} \Delta}-i \frac{2 C}{\Delta} \frac{n^{2} \alpha^{2}}{\gamma_{\perp}^{2} \Delta^{2}}\right) f_{n}\right. \\
& \left.-\tilde{y} \delta_{n, 0}-i \eta \sum_{m, j} f_{n-m} f_{j}^{*} f_{m+j}\right] .
\end{aligned}
$$

It is important to observe that the third and fourth terms in the square bracket of equation (41), which are functions of $n \alpha$, arise from the fact that the linear part of the atomic polarization (38) depends on the modal frequencies, i.e. they express the phenomenon of light dispersion. In our treatment we have kept only the linear and quadratic terms, which is in accord with the standard treatment of dispersion.

Let us now define

$$
\bar{f}_{n}(t)=f_{n}(t) \mathrm{e}^{-i n \alpha \frac{2 C}{\Delta^{2}} \frac{\kappa}{\gamma_{\perp}} t},
$$

so that equation (41) becomes

$$
\begin{aligned}
\frac{d \bar{f}_{n}}{d t}= & -\kappa\left[-\tilde{y} \delta_{n, 0}+\left(1+i \theta_{0}-i \frac{2 C}{\Delta} \frac{n^{2} \alpha^{2}}{\gamma_{\perp}^{2} \Delta^{2}}\right) \bar{f}_{n}\right. \\
& \left.-i \eta \sum_{m, j} \bar{f}_{n-m} \bar{f}_{j}^{*} \bar{f}_{m+j}\right] .
\end{aligned}
$$

Equation (43) generalizes to all modes the three-mode model derived in [32]. By combining the expression of $\tilde{F}(z, t)$ given in the equation after equations $(24 \mathrm{c})$ and $(42)$ we obtain the following expression for $\tilde{F}(z, t)$

$$
\begin{aligned}
F(z, t) & =\sum_{n} \bar{f}_{n}(t) \mathrm{e}^{-i n \alpha\left(1-\frac{2 C}{\Delta^{2}} \frac{\kappa}{\gamma_{\perp}}\right)\left(t-\frac{z}{v_{g}}\right)} \\
& =\sum_{n} \bar{f}_{n}(t) \mathrm{e}^{-i n \bar{\alpha}\left(t-\frac{z}{v_{g}}\right)},
\end{aligned}
$$

where

$$
v_{g}=\tilde{c}\left(1-\frac{2 C}{\Delta^{2}} \frac{\kappa}{\gamma_{\perp}}\right)
$$

and

$$
\bar{\alpha}=\alpha\left(1-\frac{2 C}{\Delta^{2}} \frac{\kappa}{\gamma_{\perp}}\right) .
$$

Therefore the linear dispersive correction leads to a redefinition of the light velocity $\tilde{c}$ into a group velocity $v_{g}$ as usual and a redefinition of the free spectal range from $\alpha$ to $\bar{\alpha}$. Note that $v_{g} \simeq \tilde{c}$ and $\bar{\alpha} \simeq \alpha$ because $\left(2 C / \Delta^{2}\right)\left(\kappa / \gamma_{\perp}\right)=O\left(\epsilon^{2}\right)$.

A simple glance at equation (44) shows that one can express $\tilde{F}$ as a function of $t$ and $\tau=t-z / v_{g}$ instead of $z$ and $t$. Then, by making the change of independent variables $(t, z) \longrightarrow(t, \tau)$, using the final expression in equations (44) and (43) one can check that $\tilde{F}(t, \tau)$ obeys the 
1 equation

$$
\begin{aligned}
\frac{\partial}{\partial t} \tilde{F}(t, \tau)= & -\kappa\left[-\tilde{y}+\tilde{F}(t, \tau)+i \theta_{0} \tilde{F}(t, \tau)\right. \\
& \left.+i \frac{2 C}{\gamma_{\perp}^{2} \Delta^{3}} \frac{\tilde{c}^{2}}{v_{g}^{2}} \frac{\partial^{2}}{\partial \tau^{2}} \tilde{F}(t, \tau)-i \eta|\tilde{F}(t, \tau)|^{2} \tilde{F}(t, \tau)\right]
\end{aligned}
$$

If we now introduce the normalized variables $\bar{t}=\kappa t$ as usual, and $\bar{\tau}=\sqrt{|\Delta|^{3} /(2 C)}\left(v_{g} / \tilde{c}\right) \gamma_{\perp} \tau$ we arrive at

$$
\begin{aligned}
\frac{\partial}{\partial \bar{t}} \tilde{F}(\bar{t}, \bar{\tau})= & \tilde{y}-\tilde{F}(\bar{t}, \bar{\tau})-i \theta_{0} \tilde{F}(\bar{t}, \bar{\tau}) \\
& +i \eta \frac{\partial^{2}}{\partial \bar{\tau}^{2}} \tilde{F}(\bar{t}, \bar{\tau})+i \eta|\tilde{F}(\bar{t}, \bar{\tau})|^{2} \tilde{F}(\bar{t}, \bar{\tau})
\end{aligned}
$$

which in the case $\eta=+1$ is formally identical to equation (13) for anomalous dispersion $(\bar{\eta}=-1)$ with $E$ replaced by $\tilde{F}$ and $\theta$ replaced by $\theta_{0}$. On the other hand, for $\eta=-1$ the complex conjugate of equation (48) is formally identical to equation (13), again for anomalous dispersion, with $\theta$ replaced by $-\theta_{0}$. If, instead, one uses the variable $\bar{z}=-\bar{\tau}=\gamma_{\perp} / \tilde{c} \sqrt{|\Delta|^{3} /(2 C)}\left(z-v_{g} t\right)$, equation (48) can be rephrased in the form

$$
\begin{aligned}
\frac{\partial}{\partial \bar{t}} \tilde{F}(\bar{t}, \bar{z})= & \tilde{y}-\tilde{F}(\bar{t}, \bar{z})-i \theta_{0} \tilde{F}(\bar{t}, \bar{z}) \\
& +i \eta \frac{\partial^{2}}{\partial \bar{z}^{2}} \tilde{F}(\bar{t}, \bar{z})+i \eta|\tilde{F}(\bar{t}, \bar{z})|^{2} \tilde{F}(\bar{t}, \bar{z}),
\end{aligned}
$$

where we use the variables $\bar{t}, \bar{z}$ instead of $\bar{t}, \bar{\tau}$, and equation (49) basically coincides with the longitudinal LLE formulated by ourselves in reference [31].

Finally, when the cavity is circular of radius $R$, as in the experiments which display Kerr frequency combs, we can use as a variable the angle $\varphi=\left(z-v_{g} t\right) / R=$ $\bar{z}\left(\tilde{c} / R \gamma_{\perp}\right) \sqrt{2 C /|\Delta|^{3}}$, so that the LLE can be reformulated in the form

$$
\begin{aligned}
\frac{\partial}{\partial \bar{t}} \tilde{F}(\bar{t}, \varphi)= & \tilde{y}-\tilde{F}(\bar{t}, \varphi)-i \theta_{0} \tilde{F}(\bar{t}, \varphi) \\
& +i \eta \frac{\beta}{2} \frac{\partial^{2}}{\partial \varphi^{2}} \tilde{F}(\bar{t}, \varphi)+i \eta|\tilde{F}(\bar{t}, \varphi)|^{2} \tilde{F}(\bar{t}, \varphi),
\end{aligned}
$$

with

$$
\beta=\frac{4 C \tilde{c}^{2}}{\gamma_{\perp}^{2}|\Delta|^{3} R^{2}}
$$

which basically coincides with that used in $[13,14,28,29]$.

In the general case $\gamma_{\|} \neq 2 \gamma_{\perp}$ the LLE is recovered in at least the following two opposite cases:

- When the resonant mode is dominant, so that the amplitudes $f_{n}$ for $n \neq 0$ are negligible. In this case $\tilde{F}$ becomes independent of $\tau$ and the second order derivative term in equation (47) drops.

- When the contribution of the resonant mode is negligible. In this case, by using equation (36) one arrives at an equation identical to equation (47) but with the nonlinear term multiplied by $\gamma_{\|} /\left(2 \gamma_{\perp}\right)$. The LLE in normal form (47) holds for the the field $\tilde{\tilde{F}}$ such that $\tilde{F}=\tilde{\tilde{F}} \sqrt{\gamma_{\|} /\left(2 \gamma_{\perp}\right)}$.

\section{Conclusions}

The derivation of the temporal/longitudinal LLE from the two-level Maxwell-Bloch equations, shown in Section 3, explicits in the best way the connection of the LLE itself with the multimode instability of optical bistability, previously predicted $[8,9]$ in the framework of such equations. The parametric conditions that correspond to the LLE identify an optimal configuration for the multimode instability, which becomes easily accessible experimentally. In particular, the long cavity requirement disappears and the multimode instability, which gives rise to a travelling longitudinal pattern in the cavity, can be observed even in microcavities. A point of key importance is that the four-wave-mixing process, which takes place in the Kerr medium assumed by the LLE, offers the possibility of generating broadband frequency combs, as observed in references $[12-17,22-25]$. This happens because the FWM scatters photons from the cavity mode quasi-resonant with the driving field to a number of symmetrical pairs of adjacent cavity modes (see e.g. [46]) and, next, the FWM process absorbs photons from any pair of modes (possibly, from the same mode) and generates photons in other pairs symmetrically positioned with respect to the first pair (see e.g. [30]) . The total photon momentum is preserved in the process, which thus generates a vast multimodal configuration.

The LLE provides an outstanding example of phenomena of spontaneous pattern formation that are intimately linked to a much promising applicative avenue, which has been opened by the experimental observation of broadband Kerr frequency combs [12].

\section{Author contribution statement}

Please note that you are required to include a statement which details the nature of the contribution of each author.

We thank Wulf Lange and Thorsten Ackemann for giving us permission of showing the patterns which appear in Figure 2.

\section{Appendix A: Alternative derivation of the LLE from the Maxwell-Bloch equations}

In this appendix we sketch an alternative derivation of the temporal/longitudinal LLE, which does not make use of the adiabatic elimination of the atomic variable. The derivation is actually similar to the one in Section 3, the main difference being that the atomic Bloch equations are first approximately solved in the continuum frequency domain, and then the result is inserted into the Maxwell equation for the field.

The starting point are the Maxwell-Bloch equations (16). Let us focus on the two atomic equations (16b) and $(16 \mathrm{c})$. In order to simplify the notation, we introduce the quantity

$$
H(t, z)=D(t, z)-1 \text {, }
$$



become then

$$
\begin{aligned}
\frac{\partial P}{\partial t} & =-\gamma_{\perp}[(1+i \Delta) P-F(H+1)], \\
\frac{\partial H}{\partial t} & =-\gamma_{\|}\left[\frac{1}{2}\left(F P^{*}+F^{*} P\right)+H\right] .
\end{aligned}
$$

4 Next, let us turn to the frequency domain setting

$$
X(t, z)=\int \frac{d \Omega}{\sqrt{2 \pi}} X(\Omega, z) e^{-i \Omega t},
$$

9 where

$$
\Delta(\Omega)=\frac{\omega_{A}-\left(\omega_{0}+\Omega\right)}{\gamma_{\perp}}=\Delta-\tilde{\Omega} \quad \text { with } \quad \tilde{\Omega}=\frac{\Omega}{\gamma_{\perp}}
$$

is the atomic detuning of the field component oscillating at frequency $\omega_{0}+\Omega$.

Let us assume the dispersive limit, where $|\Delta(\Omega)| \gg 1$ for all the populated frequency components of the field, i.e. the whole bandwidth of emitted light is far away from atomic resonance. In particular, we shall assume that the central frequency is far off resonance,

$$
|\Delta| \gg 1
$$

and the frequency bandwidth of the field is small compared to the central detuning

$$
|\tilde{\Omega}| \ll|\Delta| .
$$

Next, we search an approximate solution as a power expansion in series of the field amplitude $F$. Precisely, we will find a perturbative power expansion in terms of $F / \Delta$, assuming $|F / \Delta| \ll 1$. Clearly, the first order term for the polarization (the linear part of the polarization) is determined by the equation

$$
P_{L}(\Omega)=\frac{F(\Omega)}{1+i \Delta(\Omega)} .
$$

By replacing $P$ with $P_{L}$ in equation (A.6) we get a solution for the inversion correct up to second order in $F$

$$
\begin{aligned}
H_{2}(\Omega)= & -\frac{1-i \frac{\Omega}{2 \gamma_{\perp}}}{1-i \frac{\Omega}{\gamma_{\|}}} \\
& \times \int \frac{d \Omega_{1}}{\sqrt{2 \pi}} \frac{F\left(\Omega_{1}\right) F^{*}\left(\Omega_{1}-\Omega\right)}{\left[1-i \Delta\left(\Omega_{1}-\Omega\right)\right]\left[1+i \Delta\left(\Omega_{1}\right)\right]} .
\end{aligned}
$$

Finally, taking the radiative limit $2 \gamma_{\perp}=\gamma_{\|}^{2}$ and inserting this second order perturbative solution into equation (A.5) we obtain an approximate solution for the polarization, valid up to third order in $F$

$$
P(\Omega)=P_{L}(\Omega)+P_{N L}(\Omega)
$$

with

$$
\begin{aligned}
P_{N L}(\Omega)= & -\frac{1}{1+i \Delta(\Omega)} \int \frac{d \Omega_{1}}{\sqrt{2 \pi}} F\left(\Omega-\Omega_{1}\right) H_{2}\left(\Omega_{1}\right) \\
= & -\frac{1}{1+i \Delta(\Omega)} \int \frac{d \Omega_{1}}{\sqrt{2 \pi}} \\
& \times \int \frac{d \Omega_{2}}{\sqrt{2 \pi}} \frac{F\left(\Omega-\Omega_{1}\right) F\left(\Omega_{2}\right) F^{*}\left(\Omega_{2}-\Omega_{1}\right)}{\left(1-i \Delta\left(\Omega_{2}-\Omega_{1}\right)\right)\left(1+i \Delta\left(\Omega_{2}\right)\right)} .
\end{aligned}
$$

This relation can be greatly simplified by retaining only the leading order term in the dispersive limit (A.8),

$$
\begin{aligned}
P_{N L}(\Omega) \approx & \frac{i}{\Delta^{3}} \int \frac{d \Omega_{1}}{\sqrt{2 \pi}} \int \frac{d \Omega_{2}}{\sqrt{2 \pi}} \\
& \times F\left(\Omega-\Omega_{1}\right) F\left(\Omega_{2}\right) F^{*}\left(\Omega_{2}-\Omega_{1}\right),
\end{aligned}
$$

which amounts to neglecting any dispersive effect of the third order nonlinear susceptibility, i.e. assuming that $\chi^{(3)}$ depends slowly on the frequency inside the bandwidth of the light. This approximation simplifies a lot the equation, because coming back to the temporal domain, one has

$$
P_{N L}(t) \simeq \frac{i}{\Delta^{3}}|F(t)|^{2} F(t)
$$

i.e. the usual Kerr-like term for the nonlinear part of the polarization.

We now turn again our attention to the linear part of the polarization, with the aim of writing it in the temporal domain. First of all, we apply the dispersive limit (A.8) to the linear polarization

$$
\begin{aligned}
P_{L}(\Omega) & =\frac{F(\Omega)}{1+i \Delta(\Omega)}=\frac{F(\Omega)}{i \Delta} \frac{1}{1-\frac{\tilde{\Omega}}{\Delta}+\frac{1}{i \Delta}} \\
& \approx-\frac{i}{\Delta} F(\Omega)\left(1+\frac{\tilde{\Omega}}{\Delta}+\frac{\tilde{\Omega}^{2}}{\Delta^{2}} \ldots\right),
\end{aligned}
$$

where we kept only the first two leading orders in $\tilde{\Omega} / \Delta$, in order to retain in the description the effects of group velocity dispersion. We remark that, rigorously speaking, we neglected small real terms which could be on the same order of magnitude as those retained, and represent the unavoidable absorption of light. As typically done in the

$$
\begin{aligned}
& { }^{2} \text { Notice that for small enough bandwidths the radiative limit } \\
& \text { is unnecessary since } \\
& \qquad \frac{1-i \frac{\Omega}{2 \gamma_{\perp}}}{1-i \frac{\Omega}{\gamma_{\|}}} \approx 1-i \frac{\Omega}{2 \gamma_{\perp}}+i \frac{\Omega}{\gamma_{\|}}=1+i \frac{\Omega}{\gamma_{\|}}\left(1-\frac{\gamma_{\|}}{2 \gamma_{\perp}}\right) \approx 1
\end{aligned}
$$

when $|\Omega| \ll \gamma_{\perp}, \gamma_{\|}$. 
treatment of Kerr-like nonlinearity, we thus are assuming the medium is basically transparent in the frequency bandwidth of interest. Notice also that when inserting the medium inside the resonator, the frequency continuum is replaced by the discrete set of cavity modes, and with the more rigorous assumption (27) of Section 3, the expansion in equation (39) (which is the analogous of what done here) is strictly valid.

Coming back to the temporal domain

$$
\begin{aligned}
P_{L}(t) & =\int \frac{d \Omega}{\sqrt{2 \pi}} P_{L}(\Omega) e^{-i \Omega t} \\
& \approx-i \int \frac{d \Omega}{\sqrt{2 \pi}} F(\Omega) e^{-i \Omega t}\left[\frac{1}{\Delta}+\frac{\tilde{\Omega}}{\Delta^{2}}+\frac{\tilde{\Omega}^{2}}{\Delta^{3}}\right] \\
& =-\frac{i}{\Delta} F(t)+\frac{1}{\Delta^{2} \gamma_{\perp}} \frac{\partial}{\partial t} F(t)+\frac{i}{\Delta^{3} \gamma_{\perp}^{2}} \frac{\partial^{2}}{\partial t^{2}} F(t) .
\end{aligned}
$$

Inserting this result into Maxwell equation (16a) and considering again the variable $z$, we obtain

$$
\begin{aligned}
& \left(1+\frac{2 C \kappa}{\Delta^{2} \gamma_{\perp}}\right) \frac{\partial F}{\partial t}+\tilde{c} \frac{\partial F}{\partial z} \\
& =-\kappa\left[\left(1+i \theta_{0}\right) F-y+2 C P_{N L}(t)+i \frac{2 C}{\Delta^{3} \gamma_{\perp}^{2}} \frac{\partial^{2} F}{\partial t^{2}}\right]
\end{aligned}
$$

where $\theta_{0}=\theta-\frac{2 C}{\Delta}$ is the corrected cavity detuning (in accord with the definition in Eq. (19) of Sect. 3) which reflects the shift of the cavity resonances due the refractive index of the two-level medium at the reference frequency, $\kappa \theta_{0}=\omega_{c}-\frac{\kappa 2 C}{\Delta}-\omega_{0}$. This equation can be also written as

$$
\begin{aligned}
(1+ & \left.\frac{2 C \kappa}{\Delta^{2} \gamma_{\perp}}\right)\left(\frac{\partial F}{\partial t}+v_{g} \frac{\partial F}{\partial z}\right) \\
& =-\kappa\left[\left(1+i \theta_{0}\right) F-y+2 C P_{N L}(t)+i \frac{2 C}{\Delta^{3} \gamma_{\perp}^{2}} \frac{\partial^{2} F}{\partial t^{2}}\right]
\end{aligned}
$$

where

$$
v_{g}=\tilde{c}\left(1+\frac{2 C \kappa}{\Delta^{2} \gamma_{\perp}}\right)^{-1} \approx \tilde{c}\left(1-\frac{2 C \kappa}{\Delta^{2} \gamma_{\perp}}\right)
$$

is the group velocity, which coincides with the definition (45) of Section 3, once one recognizes that $\frac{2 C \kappa}{\Delta^{2} \gamma_{\perp}} \ll 1$.

The next step consists in introducing a field modal expansion as

$$
F(z, t)=\sum_{n} f_{n}(t) \mathrm{e}^{-i \Omega_{n}\left(t-\frac{z}{v_{g}}\right)},
$$

where

$$
\Omega_{n}=n \frac{2 \pi v_{g}}{\mathcal{L}}=n \bar{\alpha}
$$

are approximated expressions for the cavity resonances, which partially account for the linear propagation into the two-level medium (partially, because dispersion, i.e the quadratic term in frequency is not considered in the determination of cavity resonances). As a result, the cavity modes are equally spaced by the free spectral range $\bar{\alpha}=\frac{2 \pi v_{g}}{\mathcal{L}}$, in accord with equation (46) of Section 3 .

By substituting into equation (A.18), one than recognizes easily that in the low transmission limit $T \ll 1$ the modal amplitudes $f_{n}(t)$ have indeed a slow variation in time, because $\left|\frac{d f_{n}}{d t}\right|$ is on the order of $\left|\kappa f_{n}(t)\right|$, where $\kappa$ is the small cavity linewidth.

Considering now the dispersion term at the r.h.s of equation (A.18), we introduce the following approximation

$$
\begin{aligned}
\frac{\partial^{2} F}{\partial t^{2}} & =\sum_{n}\left[\frac{d^{2} f_{n}}{d t^{2}}-2 i \Omega_{n} \frac{d f_{n}}{d t}-\Omega_{n}^{2} f_{n}(t)\right] e^{-i \Omega_{n}\left(t-\frac{z}{v_{g}}\right)} \\
& \approx \sum_{n}-\Omega_{n}^{2} f_{n}(t) \mathrm{e}^{-i \Omega_{n}\left(t-\frac{z}{v_{g}}\right)}=\frac{\partial^{2} F}{\partial\left(z / v_{g}\right)^{2}}, \quad(\mathrm{~A} .22)
\end{aligned}
$$

where we made use of the slow variation of the $f_{n}^{\prime} \mathrm{s}$ in time,

$$
\left|\frac{d f_{n}}{d t}\right| \ll\left|\Omega_{n} f_{n}(t)\right| .
$$

Note that for the modes $n \neq 0$ (i.e. the side-bands with respect to the central frequency), this statement amounts to requiring that the cavity linewidth of each mode is much smaller than the free spectral range, which is indeed correct in the low transmission limit. For the central mode $n=0$, it amounts simply to neglecting the effects of dispersion of the group velocities inside the cavity linewidth, which is again correct in that limit. On the other side, it is worth remarking that when a large number of modes are populated, the temporal dispersion over the full bandwidth can be relevant, and it is indeed taken into account by the terms $\propto \Omega_{n}^{2} f_{n}$.

With this approximation equation (A.18) becomes

$$
\begin{aligned}
\frac{\partial F}{\partial t}+v_{g} \frac{\partial F}{\partial z}= & -\bar{\kappa}\left[\left(1+i \theta_{0}\right) F-y+2 C P_{N L}(t)\right. \\
& \left.+i \frac{2 C}{\Delta^{3} \gamma_{\perp}^{2}} \frac{\partial^{2} F}{\partial\left(z / v_{g}\right)^{2}}\right]
\end{aligned}
$$

where $\bar{\kappa}=\kappa /\left(1+\frac{2 C \kappa}{\Delta^{2} \gamma_{\perp}}\right) \approx \kappa$. Finally, we make the last cosmetic addition to equation (A.24) by introducing the change of independent variables $t^{\prime}=t, \tau=t-z / v_{g}$, which implies $\partial / \partial t=\partial / \partial t^{\prime}+\partial / \partial \tau, v_{g} \partial / \partial z=-\partial / \partial \tau$, so that equation (A.24) becomes

$$
\begin{aligned}
\frac{\partial \tilde{F}}{\partial t^{\prime}}= & -\kappa\left[\left(1+i \theta_{0}\right) \tilde{F}-\tilde{y}\right. \\
& \left.-i \eta|\tilde{F}(t)|^{2} \tilde{F}(t)+i \frac{2 C}{\Delta^{3} \gamma_{\perp}^{2}} \frac{\partial^{2} \tilde{F}}{\partial \tau^{2}}\right],
\end{aligned}
$$

where we have also inserted the explicit expression of the cubic nonlinearity obtained in equation (A.14), and the scaling (23) of the field amplitude. Clearly this equation 
coincides with equation (47) apart from the term $\propto \tilde{c}^{2} / v_{g}^{2}$ which is in any case very close to one and can be incorporated in the rescaling of the time $\tau$.

\section{References}

1. L.A. Lugiato, R. Lefever, Phys. Rev. Lett. 58, 2209 (1987)

2. A.M. Turing, Phil. Trans. R. Soc. London B 237, 37 (1952)

3. H. Haken, Synergetics: an Introduction (Springer-Verlag, Berlin, 1977)

4. G. Nicolis, I. Prigogine, Self-organization in nonequilibrium systems. From dissipative structures to order through fluctuations (Wiley, New York, 1977)

5. L.A. Lugiato, Chaos, Solitons and Fractals 4, 1251 (1994)

6. H.M. Gibbs, S.L. McCall, T.N.C. Venkatesan, Phys. Rev. Lett. 36, 1135 (1976)

7. H. Haelterman, S. Trillo, S. Wabnitz, Opt. Commun. 91 401 (1992)

8. R. Bonifacio, L.A. Lugiato, Lett. Nuovo Cim. 21, 510 (1978)

9. R. Bonifacio, M. Gronchi, L.A. Lugiato, Opt. Commun. 30, 129 (1979)

10. D.J. Jones, S.A. Diddams, J.K. Ranka, A. Stentz, R.S. Windeler, J.L. Hall, S.T. Cundiff, Science 288, 635 (2000)

11. Th. Udem, R. Holzwarth, T.W. Hänsch, Nature 416, 233 (2002)

12. P. Del'Haye, A. Schliesser, O. Arcizet, T. Wilken, R Holzwarth, T.J. Kippenberg, Nature 450, 1214 (2007)

13. Y.K. Chembo, C.R. Menyuk, Phys. Rev. A 87, 053852 (2013)

14. C. Godey, I.V. Balakireva, A. Coillet, Y.K. Chembo, Phys. Rev. A 89, 063814 (2014).

15. S. Coen, H.G. Randle, Th. Sylvestre, M. Erkintalo, Opt. Lett. 38, 37 (2013)

16. M.R.E. Lamont, Y. Okawachi, A.L. Gaeta, Opt. Lett. 38 3478 (2013)

17. A.B. Matsko, A.A. Savchenkov, W. Liang, V.S. Ilchenko, D. Seidel, L. Maleki, Opt. Lett. 36, 2845 (2011)

18. B.R. Mollow, Phys. Rev. A 5, 2217 (1972)

19. A.M. Bonch-Bruevich, V.A. Khodovoi, N.A. Chigir, Sov. Phys. J. Exp. Theor. Phys. 40, 1027 (1975)

20. F.Y. Wu, S. Exekiel, M. Ducloy, B.R. Mollow, Phys. Rev Lett. 38, 1077 (1977)

21. L.A. Lugiato, F. Prati, M. Brambilla, Nonlinear Optical Systems (Cambridge University Press, Cambridge, 2015)

22. I.S. Grudinin, L. Baumgartel, N. Yu, Opt. Expr. 20, 6604 (2012)

23. Y. Okavachi, K. Saha, J.S. Levy, Y.H. Wen, M. Lipson, A.L. Gaeta, Opt. Lett. 36, 3398 (2011)

24. T. Herr, V. Brasch, J.D. Jost, I. Mirgorodskiy, G Lihachev, M.L. Gorodetsky, T.J. Kippenberg, Phys. Rev. Lett. 113 123901, (2014)

25. W. Liang, D. Eliyahu, V. Ilchenko, A.A. Savchenkov, A.B. Matsko, D. Seidel, L. Maleki, Nat. Commun. 6, 7957 (2015)

26. S. Coen, M. Erkintalo, Opt. Lett. 38, 1790 (2013)

27. A. Coillet, Y.K. Chembo, Chaos 24, 013113 (2014)

28. J. Pfeifle, A. Coillet, R. Henriet, K. Saleh, Ph. Schindler, C. Weimann, W. Freude, I.V. Balakireva, L. Larger, Ch. Koos, Y.K. Chembo, Phys. Rev. Lett. 114, 093902 (2015)
29. J. Pfeifle, V. Brasch, M. Lauermann, Y. Yu, D. Wegner, T. Herr, K.A. Hartinger, P. Schindler, J. Li, D. Hillerkuss, R. Schmogrow, C.A. Weimann, R. Holzwarth, W. Freude, J. Leuthold, T.J. Kippenberg, C. Koos, Nat. Photon. 8, $375(2014)$

30. Y.K. Chembo, Nanophotonics 5, 214 (2016)

31. M. Brambilla, F. Castelli, A. Gatti, L.A. Lugiato, F. Prati,Instabilities and quantum noise reduction in nonlinear optical mixing, in Nonlinear Dynamics and Spatial Complexity In Optical Systems, Proceedings of the Firsty First (1992), Scottish University Summer School in Physics, edited by R.G. Harrison, J.S. Uppal, SUSSP Proceedings 41, 115 (1993)

32. M. Brambilla, F. Castelli, L.A. Lugiato, F. Prati, G. Strini, Opt. Commun. 83, 367 (1991)

33. H. Risken, K. Nummedal, J. Appl. Phys. 39, 4662 (1968)

34. R. Graham, H. Haken, Z. Phys. 213, 420 (1968)

35. L.A. Lugiato, Theory of optical bistability, Progress in Optics, Vol. XXI, edited by E. Wolf (Elsevier-North Holland, Amsterdam, 1984)

36. L.A. Lugiato, Opt. Commun. 33, 108 (1980)

37. B. Segard, B. Macke, L.A. Lugiato, F. Prati, M. Brambilla, Phys. Rev. A 39, 703 (1989)

38. I. Prigogine, R. Lefever, J. Chem. Phys. 48, 1965 (1968)

39. R. Bonifacio, L.A. Lugiato, Lett. Nuovo Cim. 21, 505 (1978)

40. K. Ikeda, Opt. Commun. 30, 257 (1979)

41. J.V. Moloney, H.M. Gibbs, Phys. Rev. Lett. 48, 1607 $(1982)$

42. D.W. McLaughlin, J.V. Moloney, A.C. Newell, Phys. Rev. Lett. 51, 75 (1985)

43. G. Nicolis, Introduction to nonlinear science (Cambridge University Press, Cambridge, 1995)

44. L.A. Lugiato, R. Lefever, Diffraction stationary patterns in passive optical systems, in Interaction of Radiation with Matter, a volume in honour of Adriano Gozzini (Quaderni della Scuola Normale Superiore, Pisa, 1987)

45. G. Grynberg, E. Le Bihan, P. Verkerk, P. Simoneau, J.R.R. Leite, D. Bloch, S. Le Boiteux, M. Ducloy, Opt. Commun. 67, 363 (1988)

46. F. Castelli, L.A. Lugiato, Phys. Rev. Lett. 68, 3284 (1992)

47. A. Gatti, E. Brambilla, L.A. Lugiato, Quantum Imaging, in Progress in Optics, Vol. LI, edited by E. Wolf (Elsevier North-Holland, Amsterdam, 2008), p. 251

48. M.I. Kolobov, Rev. Mod. Phys. 71, 1539 (1999)

49. D. Gomila, P. Colet, Phys. Rev. A 68, 011801(R) (2003)

50. D. Gomila, P. Colet, Phys. Rev. E 76, 016217 (2007)

51. P. Coullet, C. Riera, C. Tresser, Chaos 14, 193 (2004) (1994)

53. W.J. Firth, G.K. Harkness, A. Lord, J. McSloy, D. Gomila, P. Colet, J. Opt. Soc. Am. B 19, 747 (2002)

54. L.A. Lugiato, IEEE J. Quantum Electron. 39, 193 (2003)

55. Th. Ackemann, W.J. Firth, G.-L. Oppo, Fundamentals an applications of spatial dissipative solitons in photonic devices, in Advances in Atomic, Molecular and Optical Physics, edited by P.R. Berman, E. Arimondo, C.C. Lin (Elsevier North-Holland, Amsterdam, 2009), Vol. 57, p. 323

56. W.J. Firth, C.O. Weiss, Opt. Photon. News 13, 54 (2002)

57. V. Odent, M. Taki, E. Louvergneaux, New J. Phys. 13, 113026 (2011) 
58. V. Odent, M. Tlidi, M.G. Clerc, P. Glorieux, E. Louvergnaux, Phys. Rev. A 90, 011806 (R) (2014)

59. S. Barland, J.R. Tredicce, M. Brambilla, L.A. Lugiato, S. Balle, M. Giudici, T. Maggipinto, L. Spinelli, G. Tissoni, T. Knoedel, M. Miller, R. Jaeger, Nature 419, 699 (2002)

60. F. Pedaci, P. Genevet, S. Barland, M. Giudici, J.R. Tredicce, Appl. Phys. Lett. 89, 221111 (2006)

61. S. Coen, M. Haelterman, Opt. Lett. 24, 80 (1999)

62. S. Coen, M. Haelterman, Opt. Lett. 26, 39 (2001)

63. F. Leo, S. Coen, P. Kockaert, S.-P. Gorza, Ph. Emplit, M. Haelterman, Nat. Photon. 4, 471 (2010)

64. W.J. Firth, Nat. Photon. (News \& Views) 4, 415 (2010)

65. F. Leo, L. Gelens, Ph. Emplit, M. Haelterman, S. Coen, Opt. Expr. 21, 9180 (2013)
66. J.K. Jang, M. Erkintalo, S.G. Murdoch, S. Coen, Opt. Lett. 40, 4755 (2015)

67. J.K. Jang, M. Erkintalo, S. Coen, S.G. Murdoch, Nat. Commun. 6, 1 (2015)

68. M.-J. Schmidberger, D. Novoa, F. Biancalana, P.St.J. Russell, N.J. Joly, Opt. Expr. 22, 3045 (2011)

69. M. Tlidi, P. Mandel, Phys. Rev. A 59, \&2575(R) (1999)

70. S.D. Jenkins, F. Prati, L.A. Lugiato, L. Columbo, M. Brambilla, Phys. Rev. A 80, 033832 (2009)

71. A. Coillet, Y.K. Chembo, Opt. Lett. 39, 1529 (2014) 25

72. Y.K. Chembo, Phys. Rev. A 93, 033820 (2016) 26

73. A. Dutt, K. Luke, S. Manipatruni, A.L. Gaeta, P. 27 Nussenzveig, M. Lipson, Phys. Rev. Appl. 3, 044005 (2015) 28

74. F. Castelli, L.A. Lugiato, M. Vadacchino, Nuovo Cimento 29 D 10, 183 (1988) 29
30 .

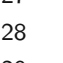

